\title{
NOISE IMMUNITY RESEARCH OF MULTI-FREQUENCY INFORMATION TRANSMISSION SYSTEM WITH CODE DIVISION OF CHANNELS
}

\section{E.A. Trubachev}

A.I. Senin trubachevea@bmstu.ru

rl1@bmstu.ru

\section{Bauman Moscow State Technical University, Moscow, Russian Federation}

\begin{abstract}
In this paper, there is noise immunity research of one version of construction multi-frequency information transmission system with code division of user's channels. An engineering method for noise immunity estimate is developed. Error probability dependences on average signal-tonoise ratio are calculated for the cases of uniform multipath intensity profile. Researchable system comparison of noise immunity with singlefrequency system of CDMA standard is made
\end{abstract}

\section{Keywords}

Simulation of random variables, code division multiple access, Rayleigh fading, multi-frequency transmission system, noise immunity, error probability, intersymbol interference

Received 08.10.2018

(c) Author(s), 2019

Introduction. The continuously increasing need for the transfer of huge amounts of information in short communication sessions is characteristic of the current development stage of science, technics and technology. Therefore, for information transmission systems (ITS) there are increased requirements for transmission speed. However, in high-speed systems when transmitting information through multipath channels, strong symbol interference with the delayed version of itself is possible, and if the extension of the channel delay exceeds the duration of the information parcel, then intersymbol interference can also reduce ITS noise immunity.

To reduce the effect of intersymbol interference and symbol interference with the delayed version of itself we can use methods of reducing the symbol rate. Common to such methods is conversion of a serial data stream into parallel streams of symbols transmitted at different subcarrier frequencies. At the same time, the duration of the information package increases proportionally, and the effect of intersymbol interference decreases. Transmitting the same symbol at different subcarrier frequencies allows you to effectively deal with channel fading. This method is implemented in multi-frequency systems with code channel separation (MC-CDMA systems) [1-6]. 
Noise Immunity Research of Multi-Frequency Information Transmission System...

MC-CDMA system block diagram. The structural diagram of an ITS for a single user is presented in Fig. 1 [2]. The data stream with the duration of information packets $T_{b}$ is converted into $P$ parallel streams. After the transformation, the duration of the parcels $T_{s}$ increases by $P$ times and becomes equal to $P T_{b}$.

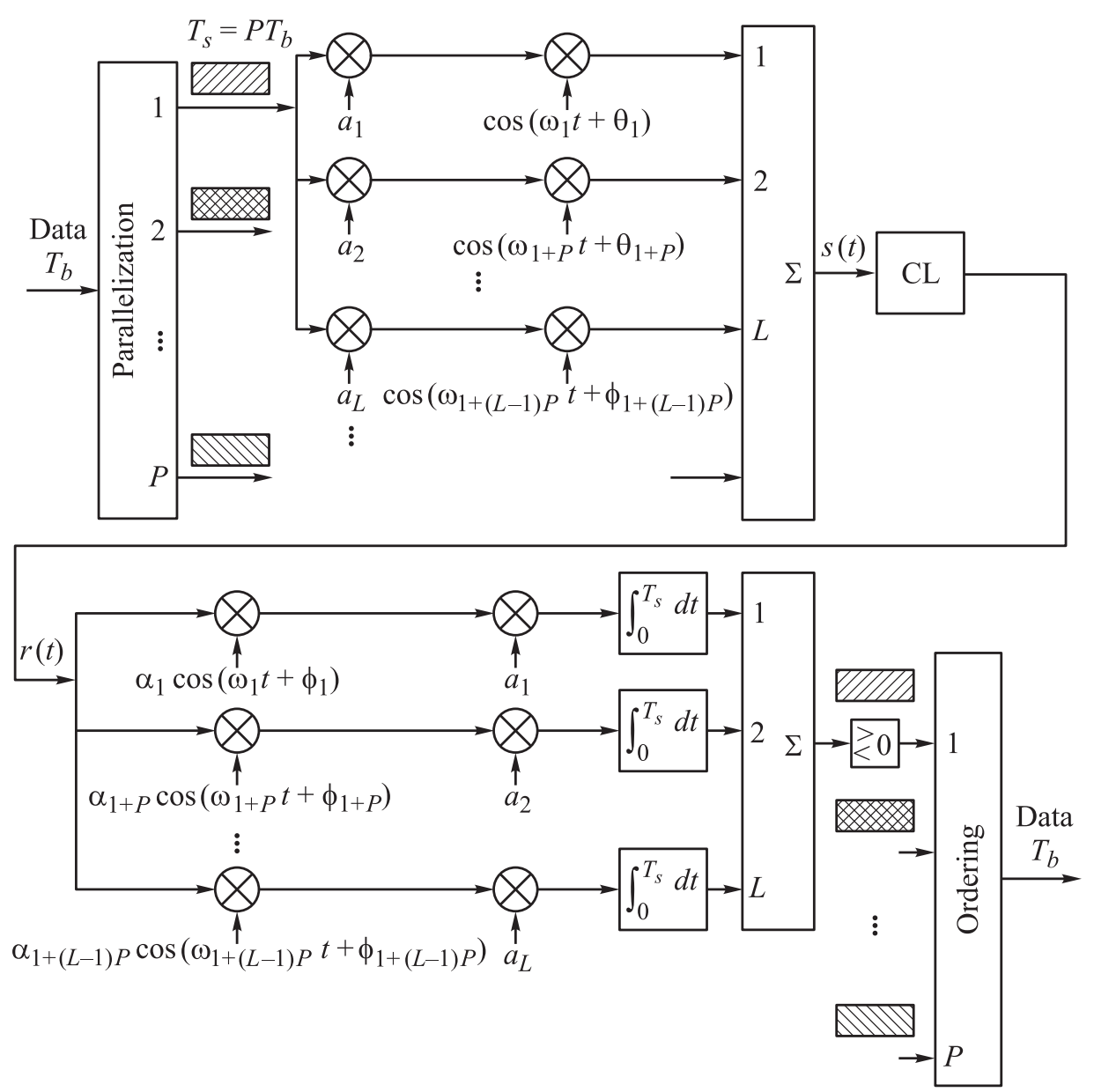

Fig. 1. Block diagram of the $M C$-CDMA system for a single user

( $\mathrm{CL}-$ communication line)

The information symbol of any $p$-th stream, $p=1,2, \ldots, P$, is transmitted over $L$ branches on $L$ subcarrier frequencies. The previously transmitted symbol in the $l$-th branch is multiplied by the $a_{l}$ symbol of the code sequence $\{c l\}$. As a code sequence, which is the address of the subscriber, a pseudo-random sequence of characters is used. Total quantity of users is $K$. Each user is assigned an individual code sequence. Thus, in the considered ITS, each user simultaneously transmits $P$ 
E.A. Trubachev, A.I. Senin

information symbols over $L$ branches. ITS uses $P L$ subcarriers in total. The generated signals are summed, forming a group signal that enters the communication line.

The subcarrier frequencies are selected from the channel signal orthogonality condition and are defined as

$$
f_{d}=f_{1}+(d-1) \frac{1}{T_{s}}, d=1,2, \ldots, P L .
$$

Frequencies assignment is carried out so that the frequency separation is maximum between the subcarriers used to transmit the same data bit. This is achieved when the symbols of the $p$-th data stream $(p=1,2, \ldots, P)$ are transmitted on $L$ subcarriers with numbers $\{p+(l-1) P, l=1,2, \ldots, L\}$. The minimum spacing in the frequency of subcarriers is $P / T_{s}$.

The receiver of the $k$-th user, $k=1,2, \ldots, K$, consists of $P$ channels. Each $p$-th channel includes $L$ branches, intended for receiving the same $p$-th information symbol. For this, following operations are performed. The received signal is multiplied by the subcarriers used to transmit the $p$-th symbol, and by chips of the $\left\{c_{l}\right\}$ code sequence. The results multiplication are integrated and summed with the weighting factors $\alpha_{k, p, l}$, which depend on the receiving method. Two cases are considered below: 1) linear addition of signals in the branches $\left.\left(\alpha_{k, p, l}=1\right) ; 2\right)$ addition of signals with weights that provide maximum signal-to-noise ratio (optimal addition). A decision on the transmitted symbol is made by comparing the weighted sum with a zero threshold. At the output of the receiving device, there is a converter of $P$ parallel data streams into one stream.

We can note that for the formation of a group signal $s_{k}(t)$ on the transmitting side there is no need to use a larger number of modulators. The group signal is obtained as a result of applying the inverse discrete Fourier transform to the signals of $P L$ branches. Accordingly, selection of channel signals at the receiving side is carried out using a direct discrete Fourier transform.

Mathematical model of the $M C-C D M A$ system. In accordance with the $M C$-CDMA block diagram (see Fig. 1), the transmitted $k$-th user signal can be represented as

$$
s_{k}(t)=\sum_{m=-\infty}^{+\infty} \sqrt{2 S_{k}} \sum_{p=1}^{P} \sum_{l=1}^{L} b_{k, p}(m) a_{k, l}(m) u_{T_{s}}\left(t-m T_{s}\right) \cos \left(\omega_{p, l} t+\theta_{k, p, l}\right)
$$

where $m$ is symbol number in the information sequence; $S_{k}$ is the signal power per one subcarrier frequency; $p$ is the channel number; $P$ is the quantity of 
Noise Immunity Research of Multi-Frequency Information Transmission System...

channels; $l$ is the branch number; $L$ is the quantity of branches in one channel; $b_{k, p}(m)$ is a sequence of information symbols in the $p$-th channel of the $k$-th user, taking value +1 or $-1 ; a_{k, l}(m)$ is the $l$-th element of the $k$-th user code sequence, taking value +1 or $-1 ; u_{T_{s}}(t)$ is a function taking value 1 for $t \in\left[0 ; T_{s}\right]$ and 0 in other cases; $T_{s}$ is the duration of a symbol transmitted in each channel; $\omega_{p, l}$ is the frequency of the subcarrier of the $l$-th branch of the $p$-th channel; $\theta_{k, p, l}(t)$ is the random phase of the subcarrier in the $l$-th branch of the $k$-th user's $p$-th channel, uniformly distributed on the interval $[0 ; 2 \pi]$.

Assuming that transmitted signals undergo Rayleigh fading with attenuation coefficients of amplitudes $\beta_{k, p, l}(t)$ and phase shifts $\varphi_{k, p, l}(t), k=1,2, \ldots, K$, $p=1,2, \ldots, P, l=1,2, \ldots, L$, uniformly distributed on an interval $[0 ; 2 \pi]$. Accordingly, the channel transfer coefficients $\mu_{k, p, l}(t)=\beta_{k, p, l}(t) e^{j \varphi_{k, p, l}(t)}$ are complex Gaussian random variables. Then, taking into account formula (1), the signal at the receiver input of this system can be represented as

$$
\begin{aligned}
r(t)= & n(t)+\sum_{m=-\infty}^{+\infty} \sqrt{2 S} \sum_{k=1}^{K} \sum_{p=1}^{P} \sum_{l=1}^{L} \beta_{k, p, l}(t) b_{k, p}(m) a_{k, l}(m) \times \\
& \times u_{T_{s}}\left(t-m T_{s}-\varsigma_{k}\right) \cos \left(\omega_{p, l} t+\phi_{k, p, l}(t)\right) .
\end{aligned}
$$

In this formula $n(t)$ is the interference (Gaussian noise with zero expectation and one-sided power spectral density $N_{0}$ ); $k$ is user number; $K$ is the quantity of users; $\beta_{k, p, l}(t)$ is the amplitude fading coefficient for the subcarrier in the $l$-th branch of the $k$-th user's $p$-th channel; $\varsigma_{k}$ is an uniformly distributed on the interval $\left[0 ; T_{s}\right]$ random variable characterizing time mismatch of the $k$-th user signal relative to the user's signal for which the noise immunity is estimated; $\varphi k, p, l(t)=\theta_{k, p, l}(t)+\varphi_{k, p, l}(t)-\omega_{p, l} \varsigma_{k}$ is a random phase of the $k$-th user signal, uniformly distributed on the interval $[0 ; 2 \pi]$. Further consideration is made for the first user.

When processing current information symbol $b_{1, p}(0)$ transmitted over the $p$-th channel, the signal at the first user correlation receiver output is determined by the expression [2]:

$$
U_{p}=V+I+J_{p}+\eta
$$

Here,

$$
V=\sqrt{\frac{S}{2}} T_{s} b_{1, p}(0) \sum_{l=1}^{L} \beta_{1, p, l}(0) \alpha_{1, p, l}
$$

is useful signal, $\alpha_{1, p, l}$ is weighting factors depending on the receiving method; 


$$
\begin{aligned}
& I=\sqrt{\frac{S}{2}} \sum_{k=2}^{K} \sum_{l=1}^{L} \alpha_{1, p, l} \beta_{k, p, l}(0) \cos \left(\phi_{k, p, l}(0)-\phi_{1, p, l}(0)\right) \times \\
& \times\left[b_{k, p}(-1) a_{k, l}(-1) a_{1, l}(0) \varsigma_{k}+b_{k, p}(0) a_{k, l}(0) a_{1, l}(0)\left(T_{s}-\varsigma_{k}\right)\right]
\end{aligned}
$$

is interference from other users signals at subcarrier frequencies that are used by the first user to transmit a symbol on the $p$-th channel, $b_{k, p}(-1)$ is previous information symbol of the $k$-th user, $a_{k, l}(-1)$ is previous element of $k$-th user code sequence;

$$
\begin{gathered}
J_{p}=\sqrt{\frac{S}{2}} \sum_{k=2}^{K} \sum_{l=1}^{L} \sum_{\substack{q=1 \\
j}}^{P} \sum_{\substack{h=1 \\
j \neq i}}^{L} \alpha_{1, p, l} \beta_{k, q, h}(0) \times \\
\times\left[\int_{0}^{\int_{k}} b_{k, q}(-1) a_{k, h}(-1) a_{1, l}(0) \cos \left(\left(\omega_{p, l}-\omega_{q, h}\right) t+\phi_{k, q, h}(0)-\phi_{1, p, l}(0)\right) d t+\right. \\
\left.+\int_{\varsigma k}^{T_{s}} b_{k, q}(0) a_{k, h}(0) a_{1, l}(0) \cos \left(\left(\omega_{p, l}-\omega_{q, h}\right) t+\phi_{k, q, h}(0)-\phi_{1, p, l}(0)\right) d t\right]
\end{gathered}
$$

is interference from the other users signals at frequencies that do not coincide with the frequencies of the first user's $p$-th channel subcarriers, $j=q+(h-1) P$, $i=p+(l-1) P$

$$
\eta=\sum_{l=1}^{L} \alpha_{1, p, l} \int_{0}^{T_{s}} n(t) a_{1, l}(0) \cos \left(\omega_{p, l} t+\phi_{1, p, l}(0)\right) d t
$$

is interference due to the influence of noise $n(t)$ at the receiver input.

Based on the central limit theorem of probability theory, the random variables $I, J_{p}, \eta$ in (2) are approximately Gaussian. Their expectations are 0 , and their variances are determined by the expressions [2]:

$$
\begin{gathered}
D_{I}=\frac{(K-1) S \sigma^{2} T_{s}^{2}}{3} \sum_{l=1}^{L} \alpha_{1, p, l}^{2} ; \\
D_{J_{p}}=\frac{(K-1) S \sigma^{2} T_{s}^{2}}{4 \pi^{2}} \sum_{l=1}^{L} \alpha_{1, p, l}^{2} \sum_{\substack{j=1 \\
j \neq p+(l-1) P}}^{P L} \frac{1}{(p+(l-1) P-j)^{2}} ; \\
D_{\eta}=\frac{N_{0} T_{s}}{4} \sum_{l=1}^{L} \alpha_{1, p, l}^{2} .
\end{gathered}
$$

The average error probability during operation time [2] is defined as

$$
P_{o}=\frac{1}{P} \sum_{p=1}^{P} \int_{0}^{+\infty} \int_{0}^{+\infty} \cdots \int_{0}^{+\infty} P_{e} W\left(\beta_{1}, \beta_{2}, \ldots, \beta_{L}\right) d \beta_{1} d \beta_{2} \cdots d \beta_{L},
$$


where

$$
P_{e}=\frac{1}{2} \Phi\left(\frac{V}{\sqrt{2 D_{U_{p}}}}\right)
$$

is error probability with fixed values of $\beta_{1}, \beta_{2}, \ldots, \beta_{L}$,

$$
\Phi(x)=\frac{2}{\sqrt{\pi}} \int_{x}^{+\infty} e^{-t^{2}} d t
$$

is error function, $V$ is useful signal at the correlation receiver output, defined by the formula (4) with $b_{1, p}(0)=1$,

$$
\begin{gathered}
D_{U_{p}}=D_{I}+D_{J_{p}}+D_{\eta}=\frac{N_{0} T_{S}}{4} \sum_{l=1}^{L} \alpha_{1, p, l}^{2}+(K-1) S \sigma^{2} T_{s}^{2} \times \\
\left.\times \frac{1}{3} \sum_{l=1}^{L} \alpha_{1, p, l}^{2}+\frac{1}{4 \pi^{2}} \sum_{l=1}^{L} \alpha_{1, p, l}^{2} \sum_{\substack{j=1 \\
j \neq p+(l-1) P}}^{P \cdot L} \frac{1}{(p+(l-1) P-j)^{2}}\right]
\end{gathered}
$$

is dispersion of the output signal $U_{p}$, defined by formula (3); $W\left(\beta_{1}, \beta_{2}, \ldots, \beta_{L}\right)$ is joint probability density of random variables $\beta_{1}, \beta_{2}, \ldots, \beta_{L}$. The Monte Carlo method was used to calculate error probability (8) in Ref. [2].

Engineering methodology for assessing $M C-C D M A$ system noise immunity. The following is an engineering methodology for assessing noise immunity. Firstly we consider the case of linear addition of signals in the receiver branches, i.e., $\alpha_{1, p, l}=1$. We enter a random variable $B=\sum_{l=1}^{L} \beta_{1, p, l}$. Then, assuming that the transmitted symbol $b_{1, p}(0)=1$, and the error probability in each $p$-th channel is the same, the average error probability can be defined as

$$
P_{o}=\int_{0}^{+\infty} \frac{1}{2} \Phi\left(\frac{\sqrt{\frac{S}{2}} T_{s} B}{\sqrt{2 D_{U_{p}}}}\right) W(B) d B,
$$

where $W(B)$ is the probability distribution density of a random variable $B$.

Integral (9) can be approximately replaced by the sum

$$
P_{o} \cong \sum_{i=1}^{Q} \frac{1}{2} \Phi\left(\frac{\sqrt{S} T_{s} i \Delta B}{2 \sqrt{D_{U_{p}}}}\right) P(i \Delta B) .
$$


E.A. Trubachev, A.I. Senin

Here, $Q$ is the ratio of a random variable $B$ maximum value, obtained in the simulation, to the value of $\Delta B$, which in the simulation was taken equal to 1 ; $P(i \Delta B)$ is the probability that the values of a random variable $B$ fall into the subinterval $((i-1) \Delta B ; i \Delta B))$. Thus, the task is reduced to modeling a random variable $B$ and finding the probabilities $P(i \Delta B), i=1,2, \ldots, Q$.

In real communication lines, fadings in channels that are spaced apart in frequency are usually correlated. This is especially true for the reviewed $M C-C D M A$ systems, in which the separation frequency of adjacent channels is small. The frequency cross-correlation function $R_{t . c}(\Delta \omega)$ of channels transmission coefficients $\mu_{k, p, l}$ is associated with the multipath intensity profile $c(\tau)$, which characterizes the dependence of the average signal power at the channel output on its delay $\tau$, by Fourier transform $[7,8]$ :

$$
R_{t . c}(\Delta \omega)=\int_{-\infty}^{\infty} c(\tau) e^{-j \Delta \omega \tau} d \tau
$$

where $\Delta \omega$ is a frequency difference of harmonic oscillations. The integral of $c(\tau)$ over a variable $\tau$ gives the full power of a signal.

In practice, uniform and exponential multipath intensity profiles are common $[2,3]$. The calculations use a uniform profile with a length of $\tau_{0}$ :

$$
c(\tau)=\left\{\begin{array}{l}
\frac{S}{\tau_{0}}, \quad 0 \leq \tau \leq \tau_{0} ; \\
0, \quad \tau \notin\left[0 ; \quad \tau_{0}\right],
\end{array}\right.
$$

where $S$ is signal power.

In the present work, the value of $\tau_{0}$ was taken to be $4 T_{c}$, where $T_{c}$ is the duration of an elementary character of a code sequence used in a singlefrequency code-division ITS ( $D S$ - CDMA), provided that the information transfer rate and frequency band occupied by the DS-CDMA system are the same as in the $M C-C D M A$ system. For the considered case $T_{c}=T_{b} / L_{1}$, where $L_{1}=60$ is the length of a DS-CDMA system code sequence [2]. The relationship between the $\tau_{0}$ and $T_{c}$ values makes it possible to compare the noise immunity of MC-CDMA and DS-CDMA systems.

Having performed the Fourier transform of $c(\tau)$ function defined by formula (11), and having carried out the normalization, we obtain an expression for a frequency normalized mutual correlation function of channels transmission coefficients

$$
r_{t . c}(\Delta \omega)=\frac{1-e^{-j \Delta \omega \tau_{0}}}{j \Delta \omega \tau_{0}} .
$$


The correlation function (12) determination is usually based on the determination of a normalized cross-correlation function $r_{\text {env }}(\Delta \omega)$ of the envelope signals in frequency channels, which coincides with great precision with a squared modulus of the function $r_{t . c}(\Delta \omega)[9,10]$ :

$$
\left|r_{t . c}(\Delta \omega)\right|^{2}=\frac{\sin ^{2}\left(\frac{\tau_{0}}{2} \Delta \omega\right)}{\left(\frac{\tau_{0}}{2} \Delta \omega\right)^{2}} .
$$

Thus,

$$
r_{e n v}(\Delta \omega) \approx\left|r_{t . c}(\Delta \omega)\right|^{2}=\frac{\sin ^{2}\left(\frac{\tau_{0}}{2} \Delta \omega\right)}{\left(\frac{\tau_{0}}{2} \Delta \omega\right)^{2}} .
$$

Considering that the signal envelope is proportional to the fading coefficient (see (2)), expression (13) will also determine the normalized frequency crosscorrelation function $r_{\beta}(\Delta \omega)$ of the fading coefficients in the channels:

$$
r_{\beta}(\Delta \omega) \approx r_{\text {env }}(\Delta \omega)=\frac{\sin ^{2}\left(\frac{\tau_{0}}{2} \Delta \omega\right)}{\left(\frac{\tau_{0}}{2} \Delta \omega\right)^{2}} .
$$

A sequence of correlated Rayleigh random numbers can be obtained using a sequence of Gaussian random numbers with zero expectation and unit variance. To do this, first a sequence of independent Gaussian random numbers is generated, using standard commands embedded in the MATLAB mathematical modeling environment. Next, a sequence of correlated Gaussian random numbers is formed, for which the method of moving summation is used.

The correlation functions of the Gaussian random process $\rho_{0}(\tau)$ and the Rayleigh random process $\rho(\tau)$, obtained from the Gaussian, are connected by the relation $\rho_{0}(\tau) \cong \sqrt{\rho(\tau)}[11]$. Therefore, to obtain a sequence of Rayleigh random numbers with a frequency correlation function (14), it is necessary, when modeling, to select a sequence of Gaussian random numbers with a normalized frequency correlation function

$$
\rho_{0}(\Delta \omega)=\frac{\sin \left(\frac{\tau_{0}}{2} \Delta \omega\right)}{\frac{\tau_{0}}{2} \Delta \omega} .
$$

ISSN 0236-3933. Вестник МГТУ им. Н.Э. Баумана. Сер. Приборостроение. 2019. № 3 
E.A. Trubachev, A.I. Senin

The sequence of Gaussian random numbers $\xi[n]$ with a correlation function (14) can be obtained in accordance with an algorithm [11]:

$$
\xi[n]=\sum_{i=-z}^{z} C_{i} x[n-i]
$$

where $z$ is the range of the moving summation;

$$
C_{i}=\frac{1}{\sqrt{\pi \frac{\gamma_{0}}{2}}} \frac{\sin \left(\frac{\gamma_{0}}{2} i\right)}{i}
$$

is moving summation coefficient, $\gamma_{0}$ is a parameter, for the reviewed case $\gamma_{0}=\tau_{0} \frac{2 \pi}{T_{b}} \approx 4 T_{c} \frac{2 \pi \cdot 2}{L T_{c}}=\frac{16 \pi}{L} ; x[n]$ is a sequence of independent Gaussian random numbers. Further, in the simulation, the value of $z$ is assumed to be 15 .

Using a sequence of correlated Gaussian random numbers, one can form a sequence of correlated Rayleigh random numbers $\zeta[n]$ :

$$
\zeta[n]=\sqrt{\xi_{1}^{2}[n]+\xi_{2}^{2}[n]}
$$

where $\xi_{1}[n], \xi_{2}[n]$ are two independent sequences of correlated Gaussian random numbers, obtained in accordance with (15).

From the obtained sequence of correlated Rayleigh random numbers one can form a random variable $B$ and find the probabilities $P(i \Delta B), i=1,2, \ldots, Q$.

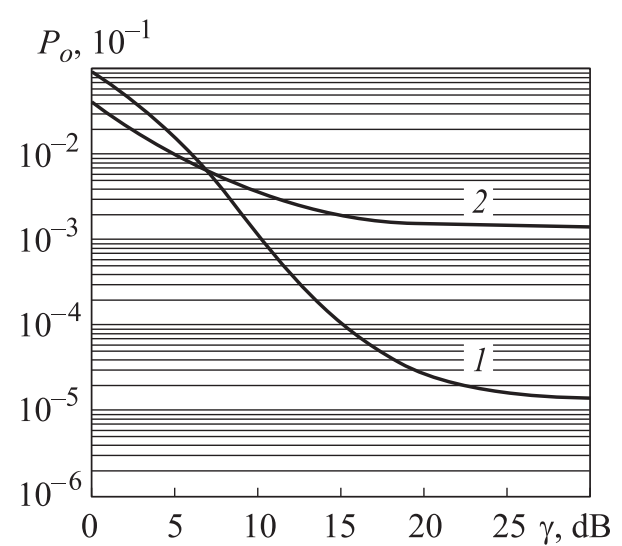

Fig. 2. Dependence of the average error probability per bit on the signalto-noise ratio for DS-CDMA (1) and $M C$-CDMA (2) systems with linear addition of signals in the branches

The average error probability per bit of information from the signal-to-noise ratio $\gamma=\frac{S T_{s}}{N_{0} L} \mathrm{M}\left\{B^{2}\right\}$ is found by the formula (10). The dependence of the average error probability per bit on the signal-to-noise ratio for $K=10, P=4$, $L=119$ (curve 1) is shown in Fig. 2. The same figure shows the dependence, taken from [2], for the DS-CDMA system (curve 2) for $K=10, L_{1}=60$.

The $M C$-CDMA system has greater noise immunity compared to the $D S-C D M A$ system (see Fig. 2). With a signal-to-noise ratio of $20 \mathrm{~dB}$, the aver- 
age probability error per bit for the $M C$-CDMA system is $\sim 0,3 \cdot 10^{-4}$, for the DS-CDMA system $-\sim 0,2 \cdot 10^{-2}$.

Note that, based on the central limit theorem of the probability theory, the distribution of a random variable $B$ obtained in the simulation is close in form to the Gaussian law (Fig. 3). Therefore, when calculating the error probability, you can directly use the formula (9). The expectation and variance of the Gaussian probability density distribution are found from the distribution of a random variable $B$, obtained by simulation.

The dependence of the average error probability per bit of information on the signal-to-noise ratio when using simulation and approximation of a random variable $B$ distribution by the Gaussian law for $K=10, P=4, L=119$ is shown in Fig. 4.

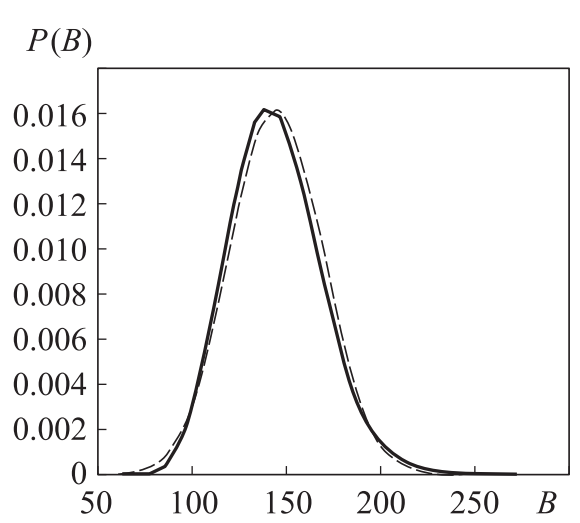

Fig. 3. The distribution of a random variable $B$ (solid line) and its approximation by the Gaussian distribution law (dashed line) at $L=119$

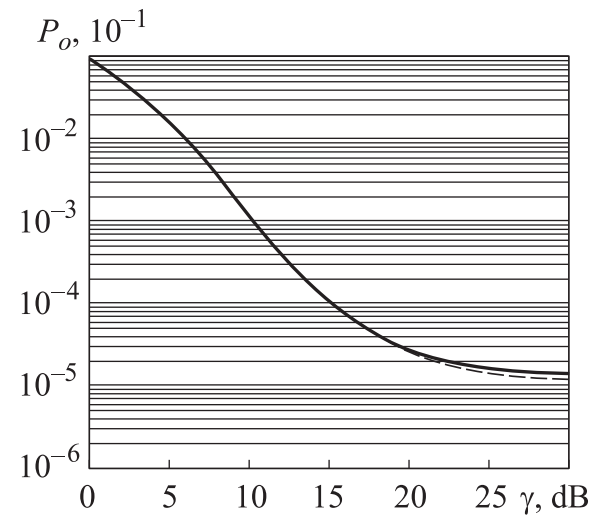

Fig. 4. The dependence of the average error probability per bit on the signal-to-noise ratio for the $M C-C D M A$ system using simulation (solid line) and approximation of a random variable $B$ distribution by the

Gaussian law (dashed line) at $L=119$

The use of a Gaussian approximation (see Fig. 4) of distribution gives results that differ slightly from the results obtained using formula (10).

With optimal addition of signals in branches with weighting coefficients $\alpha_{1, p, l}=\beta_{1, p, l}$, providing the maximum value of the signal-to-noise ratio, the useful signal $V$ at the correlation receiver output taking into account (4) with $b_{1, p}(0)=1$ is determined by the formula

$$
V=\sqrt{\frac{S}{2}} T_{s} \sum_{l=1}^{L} \beta_{1, p, l}^{2}=\sqrt{\frac{S}{2}} T_{s} B^{\prime},
$$

and the interference variance, taking into account expressions (5)-(7) determined by the formula 


$$
\begin{gathered}
D_{U_{p}}=\frac{N_{0} T_{s}}{4} B^{\prime}+(K-1) S \sigma^{2} T_{s}^{2} \times \\
\times\left[\frac{1}{3} B^{\prime}+\frac{1}{4 \pi^{2}} \sum_{l=1}^{L} \beta_{l}^{2} \sum_{\substack{j=1 \\
j \neq p+(l-1) P}}^{P L} \frac{1}{(p+(l-1) P-j)^{2}}\right] .
\end{gathered}
$$

The nested sum $\sum_{\substack{j=1 \\ j \neq p+(l-1) P}}^{P L} \frac{1}{(p+(l-1) P-j)^{2}}$ in expression (17) is practically independent of the parameter $l$ of the outer sum. In particular, for $L=119$, it is approximately equal to 3.25 (Fig. 5). This allows us to simplify the expression (17) to the form

$$
D_{U_{p}} \cong\left[\frac{N_{0} T_{s}}{4}+(K-1) S \sigma^{2} T_{s}^{2}\left[\frac{1}{3}+\frac{3,25}{4 \pi^{2}}\right]\right] B^{\prime} .
$$

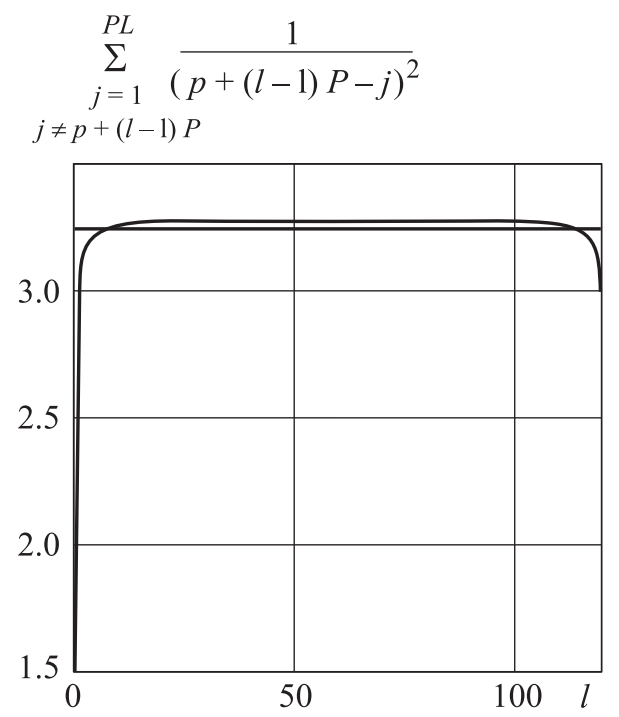

Fig. 5. The dependence of the nested sum value on the outer sum parameter $l$ at $L=119$

Taking into account (16) and (18), we can define the average error probability as

$$
P_{o}=\int_{0}^{\infty} \frac{1}{2} \Phi\left(\frac{\sqrt{\frac{S}{2}} T_{s} B^{\prime}}{\sqrt{2 D_{U_{p}}}}\right) W\left(B^{\prime}\right) d B^{\prime} \cong \sum_{i=1}^{Q^{\prime}} \frac{1}{2} \Phi\left(\frac{\sqrt{S} T_{s} i \Delta B^{\prime}}{2 \sqrt{D_{U_{p}}}}\right) P\left(i \Delta B^{\prime}\right),
$$

where $Q^{\prime}$ is determined by analogy with $Q$ in the formula (10). 
Noise Immunity Research of Multi-Frequency Information Transmission System...

Therefore, the problem solution for the case of optimal signals addition in the branches is similar to the problem solution for the case of linear addition and reduces to modeling a random variable $B^{\prime}$ and finding the probabilities $P\left(i \Delta B^{\prime}\right), i=1,2, \ldots, Q^{\prime}$.

The dependence of the average error probability per bit of information on the signal-to-noise ratio for the optimal addition scheme for $K=10, P=4$, $L=119$ is shown in Fig. 6 (curve 1). The same figure shows a similar dependence for the linear addition scheme (curve 2).

When the error probability per bit is $10^{-4}$, the energy gain of the optimal

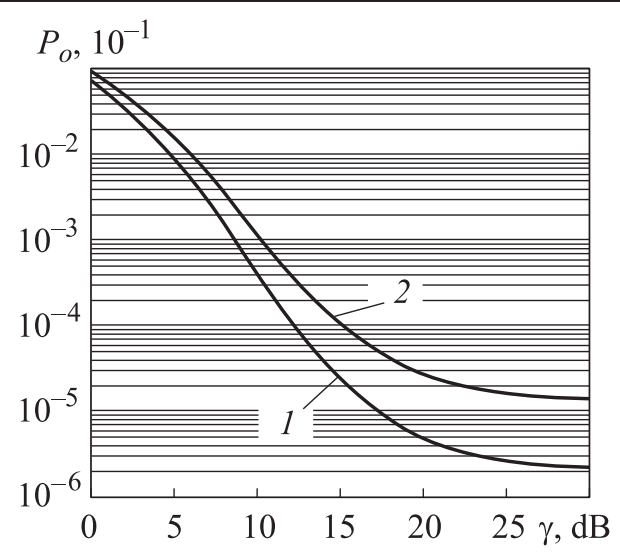

Fig. 6. The dependence of the average error probability per bit on the signalto-noise ratio for the $M C$-CDMA system using optimal (1) and linear (2) signal addition circuits in the branches at $L=119$ addition scheme as compared to the linear addition scheme is $\sim 2.5 \mathrm{~dB}$.

Conclusion. An engineering method for calculating the noise immunity of the MC-CDMA system with linear and optimal signal addition schemes in the receiver branches under Rayleigh fading is presented. It is shown that the $M C-C D M A$ system has greater noise immunity than the single-frequency DS-CDMA system, all other conditions being the same. Thus, with an average signal-to-noise ratio of $20 \mathrm{~dB}$, the $M C$-CDMA system with $K=10, P=4, L=119$ provides an error probability of $\sim 0,3 \cdot 10^{-4}$, and a single-frequency DS-CDMA system $\sim 0,2 \cdot 10^{-2}$.

In the linear signals addition scheme using the Gaussian approximation of the fading coefficients sum distribution in the branches, the calculation results of the $M C-C D M A$ system noise immunity differ slightly from the case of using the distribution obtained by simulation.

The optimal scheme of signals addition in the branches with $K=10, P=4$, $L=119$ allows to obtain an energy gain of $\sim 2.5 \mathrm{~dB}$ with an error probability of $10^{-4}$ compared to the linear addition scheme.

The presented engineering methodology for assessing the MC-CDMA system noise immunity is easy to use, and the obtained results do not practically differ from those obtained by a more accurate method.

Translated by D. Babikova 
E.A. Trubachev, A.I. Senin

\section{REFERENCES}

[1] Fazel K., Kaiser S. Multi-carrier and spread spectrum systems: from OFDM and MC-CDMA to LTE and WiMAX. Singapore, Markono Print Media, 2008.

[2] Gui X., Ng T.S. Performance of asynchronous orthogonal multicarrier CDMA system in frequency selective fading channel. IEEE Trans. Commun., 1999, vol. 47, no. 7, pp. 1084-1091. DOI: 10.1109/26.774858

[3] Sourour E.A., Nakagawa M. Performance of orthogonal multicarrier CDMA in a multipath fading channel. IEEE Trans. Commun., 1996, vol. 44, no. 3, pp. 356-367. DOI: $10.1109 / 26.486330$

[4] Hara S., Prasad R. Design and performance of multicarrier CDMA system in frequency-selective Rayleigh fading channels. IEEE Trans. Vehic. Technol., 1999, vol. 48, no. 5, pp. 1584-1595. DOI: 10.1109/25.790535

[5] Hanzo L., Münster M., Choi B.J., et al. OFDM and MC-CDMA for broadband multi-user communications, WLANs and broadcasting. John Wiley \& Sons, 2003.

[6] Linnartz J.-P.M.G. Performance analysis of synchronous MC-CDMA in mobile Rayleigh channel with both delay and Doppler spreads. IEEE Trans. Vehic. Technol., 2001, vol. 50, no. 6, pp. 1375-1387. DOI: $10.1109 / 25.966570$

[7] Proakis J. Digital communications. McGraw-Hill, 2000.

[8] Lee W.C.Y. Mobile communications engineering: theory and applications. McGraw-Hill, 1997.

[9] Levin B.R. Teoreticheskie osnovy statisticheskoy radiotekhniki. T. 1 [Theoretical foundations of statistical radio engineering. Vol. 1]. Moscow, Sovetskoe radio Publ., 1966.

[10] Stein S., Jones J.J. Modern communication principles: with application to digital signaling. McGraw-Hill, 1967.

[11] Bykov V.V. Tsifrovoe modelirovanie $\mathrm{v}$ statisticheskoy radiotekhnike [Digital modelling in statistical radio engineering]. Moscow, Sovetskoe radio Publ., 1971.

Trubachev E.A. - Post-Graduate Student, Assistant, Department of Radio Electronic Systems and Devices, Bauman Moscow State Technical University (2-ya Baumanskaya ul. 5, str. 1, Moscow, 105005 Russian Federation).

Senin A.I. - Cand. Sc. (Eng.), Assoc. Professor, Department of Radio Electronic Systems and Devices, Bauman Moscow State Technical University (2-ya Baumanskaya ul. 5, str. 1, Moscow, 105005 Russian Federation).

\section{Please cite this article as:}

Trubachev E.A., Senin A.I. Noise immunity research of multi-frequency information transmission system with code division of channels. Herald of the Bauman Moscow State Technical University, Series Instrument Engineering, 2019, no. 3, pp. 14-26.

DOI: 10.18698/0236-3933-2019-3-14-26

26 ISSN 0236-3933. Вестник МГТУ им. Н.Э. Баумана. Сер. Приборостроение. 2019. № 3 